\title{
Lymph Node Hilum
}

National Cancer Institute

\section{Source}

National Cancer Institute. Lymph Node Hilum. NCI Thesaurus. Code C33031.

The concave side of the lymph node. 